\title{
INCIDENTAL CHEST RADIOGRAPHIC FINDINGS IN HEALTHY INDIAN RESEARCH SUBJECTS
}

\author{
Deepa Rasaily1, Abhinav Jain², Rajinder K. Jalali ${ }^{3}$
}

${ }^{1}$ Senior Research Manager, Clinical Pharmacology Unit, Department of Medical Affairs and Clinical Research, Sun Pharmaceutical Industries Ltd., New Delhi.

${ }^{2}$ Assistant Professor, Department of Radio-diagnosis, Hamdard Institute of Medical Sciences and Research, New Delhi, India.

3 Vice President and Head, Department of Medical Affairs and Clinical Research, Sun Pharmaceutical Industries Ltd., Gurgaon, Haryana.

\section{ABSTRACT}

\section{BACKGROUND}

Chest radiographs, regardless of clinical indications may produce certain incidental findings (IF) which may have consequences for patients and also need of further evaluation. Such incidental findings have risks and benefits associated with them.

The objective of this study was to estimate the frequency of IFs in chest radiographs of healthy research volunteers enrolled in a single research centre who were undergoing screening procedure for participation in clinical studies.

\section{MATERIALS AND METHODS}

1704 volunteers enrolled in the volunteer database underwent baseline screening procedure to determine their eligibility for the purpose of participation in healthy volunteers' research studies. 1408 participants with normal or clinically insignificant findings on baseline screening had undergone chest X-ray (CXR) and laboratory tests. All reported chest radiographic findings were defined as incidental.

\section{RESULTS}

Out of 1408 CXRs obtained, at least one IF was reported in 197 radiographs, more than one was reported in 13 radiographs in a total of $224(15.91 \%)$ incidental findings. $5.12 \%$ of incidental findings were considered to be clinically relevant; $3.69 \%$ probably relevant and $7.1 \%$ were not clinically relevant.

\section{CONCLUSION}

Incidental findings in healthy volunteers are common in research. Researchers have an ethical obligation to research participants to identify and assess the potential for IFs and disclosure of such findings. The continued development of protocols for managing IFs in research and the establishment of guidelines are needed to ensure that research procedures reflect the best interest of participants.

\section{KEYWORDS}

Incidental Findings, Chest X-ray, Healthy Volunteers, Research Study.

HOW TO CITE THIS ARTICLE: Rasaily D, Jain A, Jalali RK. Incidental chest radiographic findings in healthy Indian research subjects. J. Evolution Med. Dent. Sci. 2017;6(14):1115-1119, DOI: 10.14260/Jemds/2017/242

\section{BACKGROUND}

Chest X-ray is one of the most common examinations performed for evaluation of thoracic diseases. Chest radiographs, regardless of clinical indications, may not only provide information relevant to the acute illness, but may also yield certain incidental findings which may have consequences for patients and need of further evaluation.1-4

Incidental findings in healthy volunteers during research procedure are common. ${ }^{5}$ In chest radiography, these findings may range from insignificant findings like cervical ribs to significant findings needing immediate medical attention like Pulmonary Koch's.

Financial or Other, Competing Interest: None.

Submission 28-10-2016, Peer Review 02-02-2017,

Acceptance 08-02-2017, Published 16-02-2017.

Corresponding Author:

Dr. Abhinav Jain,

Assistant Professor

Department of Radio-diagnosis,

Hamdard Institute of Medical

Sciences and Research,

New Delhi-110062,

India.

E-mail: drabhinavjain@gmail.com

DOI: $10.14260 /$ jemds $/ 2017 / 242$
These incidental findings have risks and benefits associated with them. Some studies show that early identification of an incidental finding might be of benefit, particularly if the finding is treatable and early diagnosis improves the disease outcome. ${ }^{5}$ For instance, most of the pulmonary nodules are incidentally detected on chest X-rays (CXRs) or CT scans. It is estimated that $0.09 \%$ to $0.2 \%$ of all CXR will incidentally detect pulmonary nodules. ${ }^{6}$ In CT angiograms obtained to diagnose pulmonary embolism, 13\% of cases in a study were reported to have incidental findings of pulmonary nodules. ${ }^{7}$ While the differential diagnosis of lung nodules includes several benign diseases, the prevalence of malignancy in patients with pulmonary nodules ranges from $1.1 \%$ to $12 \% .{ }^{8}$ However, on the other hand, discovery of an incidental finding with unknown significance may trigger anxiety, burdens and the costs of further investigations to rule out a clinical finding which may turn out to be benign.

An incidental finding (IF) in a healthy volunteer in research is a "finding that concerns an individual research subject which has potential health or reproductive importance and is discovered during the course of conducting research but is beyond the aims of the study". ${ }^{9}$ Such findings may not be anticipated in the research study. Research IFs may also arise while collecting baseline physiological information in determining whether a potential volunteer is eligible for inclusion in the research study. ${ }^{9}$ 
IFs found in healthy volunteers during research have important implications, particularly in the areas of informed consent, subjects' rights, clinical data analysis and handling of such findings. ${ }^{5}$

The objective of this study was to estimate the frequency of IFs in chest radiographs of healthy research volunteers enrolled in a single research centre who were undergoing screening procedure for participation in clinical studies.

\section{MATERIALS AND METHODS}

The screening protocol was approved by the local Institutional Ethics Committee (IEC). The study was conducted in Clinical Pharmacology Unit, Sun Pharmaceutical Industries Ltd., HAH Centenary Hospital, Delhi. The study conformed to the ethical guidelines of Declaration of Helsinki and the principles of Good Clinical Practice.

The study was conducted as a non-randomised screening procedure of volunteers enrolled in the volunteer database in a single research unit to determine their eligibility for the purpose of participation in healthy volunteers' research studies, primarily bioequivalence studies.

A written informed consent was obtained from all the participants for the screening procedure including noninvasive investigations. Participants were informed of any foreseeable risks associated with research participation during informed consent process.

The protocol consisted of baseline screening procedure which included recording of demographic data, history of smoking and alcohol, vital signs (Sitting blood pressure, pulse rate, oral temperature), 12-lead electrocardiogram (ECG), medical history and physical examination. Participants with normal or clinically insignificant findings on baseline screening were followed by chest X-ray (PA view) and laboratory test including haematology, biochemistry, serology and routine/microscopic urinalysis. Participants were then considered eligible to participate in healthy volunteers' research studies based on CXR findings and acceptable laboratory parameters.

1704 volunteers enrolled in the volunteer database underwent baseline screening procedure which extended from November 2015 to March 2016. A total of 1408 volunteers with normal or clinically insignificant findings on baseline screening had undergone CXR and laboratory test. The inclusion criteria were male volunteers, in the age range of 18-45 years and within $20 \%$ of their ideal body weight who were in generally good health and be able to read, sign and date an informed consent document. Exclusion criteria included exposure to any investigational products in the last three months, difficulty in abstaining from smoking or alcohol during study participation, history of blood donation in the last three months and a history or current condition of gastrointestinal, cardiovascular, hepatic, renal or respiratory disease.

All the radiographs were carried out at department of Radiodiagnosis, of the institute, using Siemens MultiX DR digital radiography system as per standard departmental protocol. The kVp used was approx. 125 and MAS 2-3. The digital radiographic images obtained were assessed on Picture Archiving and Communication System (PACS) using certified medical grade imaging monitors. A hard copy of all the images was saved for documentation purpose. Each radiograph was reported by two experienced radiologists and only the findings reported by both were accepted.

\section{RESULTS}

All the participants were Asian (Indian) males. The mean age of the participants was $29.17( \pm 6.32)$ and body mass index (BMI) was $21.52( \pm 2.65)$. All the participants were from in and around Delhi, India. Most of the participants were from lower socioeconomic status. The characteristics of the participants involved in the study are reported in table 1.

All reported findings were defined as incidental and their clinical relevance was based on literature review and clinical judgment.

Out of 1408 CXRs obtained, at least one IF was reported in 197 radiographs, more than one was reported in 13 radiographs in a total of $224(15.91 \%)$ IFs. Of the total radiographs, $5.12 \%$ IFs were considered to be clinically relevant; $3.69 \%$ probably relevant and $7.1 \%$ clinically not relevant. The most common clinically relevant finding was features suggestive of possible active tuberculosis (nodules with consolidation, cavitation, collapse, atelectasis and/or lymphadenopathy) which were reported in $2.98 \%(\mathrm{n}=42)$ of total radiographs. Nodules were reported in $1.14 \%(n=16)$ and solitary pulmonary nodule (SPN) in $0.50 \%(n=7)$ of total radiographs. Pleural effusion was reported in $0.36 \%(n=5)$ and mild cardiomegaly in $0.14 \%(\mathrm{n}=2)$.

At least two signs of chronic obstructive pulmonary disease (hyperinflation, flattened diaphragm, tubular cardiac silhouette, rounded costophrenic angle, prominent bronchovascular markings) were reported in $2.06 \%(n=29)$ of total radiographs and was the most common probably relevant IF.

Of the non-relevant findings, scoliosis was the most common finding $(1.28 \%)$. Percentages of IFs are reported in Table 2 and 3.

\begin{tabular}{|c|c|}
\hline Age (Mean \pm SD) & $\mathbf{2 9 . 1 7}(\mathbf{\pm 6 . 3 2 )}$ \\
\hline \multicolumn{2}{|c|}{ Age Group; n (\%) } \\
\hline $18-20$ & $109(7.74)$ \\
\hline $30-29$ & $656(46.60)$ \\
\hline $40-45$ & $542(38.49)$ \\
\hline \multicolumn{2}{|c|}{ Gender (\%) } \\
\hline Male & $101(7.17)$ \\
\hline BMI (Mean \pm SD) & $21.52( \pm 2.65)$ \\
\hline Race/Ethnicity; n (\%) \\
\hline \multicolumn{2}{|c|}{ Smoking Behaviour; n (\%) } \\
\hline Smokers & $436(30.97)$ \\
\hline Ocian (100\%) \\
\hline Non-Smokers & $972(69.03)$ \\
\hline Factory Workers & $789(56.04)$ \\
\hline Non-Factory Workers & $531(37.71)$ \\
\hline Unemployed & $88(6.25)$ \\
\hline Table 1. Characteristics of the Participants (n=1408) \\
\hline
\end{tabular}




\begin{tabular}{|c|c|c|}
\hline \multirow{3}{*}{$\begin{array}{c}\text { Clinical } \\
\text { Relevance }\end{array}$} & $\begin{array}{l}\text { No. of Incidental } \\
\text { Findings } \\
\end{array}$ & $\begin{array}{c}\text { No. of } \\
\text { Radiographs } \\
\end{array}$ \\
\hline & $\mathrm{n}=224^{\mathrm{a}}$ & $\mathrm{n}=1408$ \\
\hline & $\mathrm{N}(\%)$ & $\%$ \\
\hline \multicolumn{3}{|c|}{ Relevant (n= 72) } \\
\hline $\begin{array}{l}\text { Findings Suggestive } \\
\text { of Possible Active TB }\end{array}$ & $42(18.75)$ & 2.98 \\
\hline Nodules & $16(7.14)$ & 1.14 \\
\hline SPN & $7(3.13)$ & 0.50 \\
\hline Pleural Effusion & $5(2.23)$ & 0.36 \\
\hline Cardiomegaly & $2(0.89)$ & 0.14 \\
\hline \multicolumn{3}{|c|}{ Probably Relevant $(n=52)$} \\
\hline Signs of COPD & $29(12.95)$ & 2.06 \\
\hline $\begin{array}{l}\text { Blunt Costophrenic } \\
\text { Angle }\end{array}$ & $23(10.27)$ & 1.63 \\
\hline \multicolumn{3}{|c|}{ Not Relevant $(n=100)$} \\
\hline Scoliosis (Mild) & $18(8.04)$ & 1.28 \\
\hline Fibrosis & $19(8.48)$ & 1.35 \\
\hline Pleural Thickening & $16(7.14)$ & 1.14 \\
\hline Cervical Ribb & $13(5.80)$ & 0.92 \\
\hline Calcified Nodules & $10(4.46)$ & 0.71 \\
\hline Azygos Fissure & $4(1.79)$ & 0.28 \\
\hline Others ${ }^{c}$ & $20(8.93)$ & 1.42 \\
\hline
\end{tabular}

aReported in 210 radiographs.

bBilateral $=4$, Unilateral $=9(\mathrm{Rt}>\mathrm{Lt})$.

cRib anomalies $=7$, Spina bifida $=3$, Spinal deformity $=2$, Old

fracture $=4$, Right sided aortic arch $=1$, calcification of aortic

knuckle $=1$, prominent cardiophrenic fat pad $=1$, unfolding

of aorta $=1$.

\begin{tabular}{|c|c|c|c|c|}
\hline Variables & Age (Mean \pm SD) & BMI (Mean \pm SD) & Smokers N (\%) & Non-smokers N (\%) \\
\hline Total No. of Volunteers (n=1408) & $29.17( \pm 6.32)$ & $21.52( \pm 2.65)$ & $436(30.96)$ & $972(69.03)$ \\
\hline $\begin{array}{c}\text { CXRs with } \\
\text { at least 1 IF (n=210) }\end{array}$ & $31.37( \pm 6.65)$ & $20.78( \pm 2.60)$ & $80(18.34)$ & $130(13.37)$ \\
\hline $\begin{array}{c}\text { Findings Suggestive } \\
\text { Cf Possible Active TB (n=42) }\end{array}$ & $29.43 \pm 7.29$ & $20.00 \pm 2.28$ & $25(59.52)$ & $17(40.48)$ \\
\hline Nodules (n=16) & $32.13 \pm 6.8$ & $22.19 \pm 2.3$ & $10(62.5)$ & $6(37.5)$ \\
\hline SPN (n=7) & $34.71 \pm 5.31$ & $20.02 \pm 2.68$ & $5(71.43)$ & $2(28.57)$ \\
\hline Pleural Effusion (n=5) & $27.2 \pm 7.43$ & $20.28 \pm 1.57$ & 0 & $5(100)$ \\
\hline Cardiomegaly (n=2) & $31.5 \pm 0.71$ & $23.35 \pm 4.45$ & 0 & $2(100)$ \\
\hline \multicolumn{7}{|c|}{ Probably Relevant Findings (n=52) } & & $11(37.93)$ \\
\hline Signs of COPD (n=29) & $34.48 \pm 5.07$ & $19.08 \pm 1.57$ & $18(62.07)$ & $11(47.83)$ \\
\hline Blunt Costophrenic Angle (n=23) & $29.09 \pm 6.12$ & $21.3 \pm 2.56$ & $12(52.17)$ & \\
\hline
\end{tabular}

\section{DISCUSSION}

In the present study, 1408 healthy volunteers underwent CXR as a part of screening procedure for participation in clinical studies. The prevalence of IFs was $15.91 \%$ while $5.12 \%$ of volunteers had clinically relevant findings.

Review of literature does not yield many studies discussing the IFs on chest radiographs in healthy population. MRI research has yielded the most explicit discussion of IFs. This could be because MRI is more commonly used to study normal population due to its signal characteristics and lack of ionising radiation as compared to other imaging modalities. ${ }^{9}$

Prevalence of IFs varies in each research domain and may be influenced by the study population, research methodology and the imaging modality used. Based on a published metaanalysis, prevalence of IFs on brain MRI was found to be $2.7 \%{ }^{10}$ and with body MRI, the prevalence was $12.8 \% .{ }^{11}$ In a review of chest CT screening studies, $7.7 \%$ of patients who underwent screening for coronary artery disease and 14.2\% of patients undergoing screening for lung cancer had IFs that required additional follow-up. ${ }^{12}$

A study on IFs in adult patients with acute cough found $19 \%$ IFs and $3 \%$ clinically relevant findings on chest radiographs. ${ }^{13}$ However, this study included only those patients who had presented with complaint of cough and not healthy individuals. Out of the reported IFs in the published study, $23.7 \%$ of signs suggesting asthma and COPD have been reported $^{13}$ as compared to $12.95 \%$ in this study. The differences reported in the findings of the current study and the published study could be explained due to differences in mean age between the participants of both studies and also the symptomatic presentation in the published study. Also, $44.37 \%$ of IFs were reported in the published study to be probably not relevant which compares with $44.64 \%$ of nonrelevant findings in this study.

Though IFs are frequently reported, only a small percentage of findings may be clinically relevant. Orme and 
colleagues indicate that the evaluation of imaging completed for research purposes leads to a high number of IFs that may result in important health benefits to a small but significant minority of participants. In the present study, 5.12\% of IFs have been considered to be clinically relevant and the most common finding was features suggestive of possible active tuberculosis (TB). According to a joint statement issued by the American Thoracic Society (ATS) and the Centre for Disease Control and Prevention, ${ }^{14}$ persons infected with $\mathrm{M}$. tuberculosis, as demonstrated by a positive tuberculin skin test, should be classified on the basis of clinical, bacteriologic and radiographic evaluation into one of the following three categories.

a. TB infection, no disease.

b. TB infection, clinically active; and.

c. TB infection, clinically inactive.

Since the status of tuberculin skin test and bacteriologic examination were not known in these participants, disease activity could not be determined. Many people with active TB do not experience classic symptoms in the early stages of the disease. Moreover, the clinical signs and symptoms of pulmonary TB in an infected adult are often non-specific and approximately $5 \%$ of active adult cases may show complete absence of symptoms. ${ }^{15}$ Since all the radiographic findings were assessed in isolation, findings suggestive of possible active tuberculosis have been classified as clinically relevant. Also, the present study comprised of healthy adult volunteers and there is a possibility that volunteers with subclinical complaints might not have disclosed their symptoms.

Pulmonary nodules may be solitary or multiple. Most of the pulmonary nodules are incidentally detected. In one of the lung cancer screening trials, $7 \%$ of CXR scans obtained from previously healthy individuals showed pulmonary nodules. ${ }^{8}$ Of the total radiographs, nodules and SPN was reported to be $1.14 \%$ and $0.50 \%$ respectively in this study. The current study; however, included only asymptomatic adult population.

Signs of COPD were observed in $2.06 \%$ of radiographs. Though this finding was observed more in smokers, 11 $(37.93 \%)$ of the volunteers with signs of COPD were nonsmokers. Smoking is particularly an important factor for COPD, but occupational exposures to dusts, environmental air pollution, gases and fumes, and certain other factors also play a role in the pathogenesis of COPD. ${ }^{16}$ Becklakein in 1989, concluded that occupational exposure to dust and/or to dust and fumes may have a causal link to the pathogenesis of COPD. ${ }^{17}$ In 2003, ATS published a review of the evidence of the association of occupational factors in the pathogenesis of obstructive airway diseases and quantified the contribution of work related risk to the burden of these diseases in the general population. Based on the results it was concluded that about $15 \%$ of COPD cases could be attributable to workplace exposure. ${ }^{18}$ The findings of the ATS review was further confirmed by two reviews by Blanc and Torén ${ }^{19}$ and the Norwegian Medical Association in 2007. In the present study, signs of COPD in non-smokers can be attributed to occupational exposures as most of the volunteers were factory workers.

IFs in healthy volunteers during research study are common. ${ }^{5}$ Researchers have an ethical obligation to research participants to identify and assess the potential for IFs and disclosure of such findings. Procedures for disclosure and management of IFs should be clearly communicated to the participants during Informed consent process. Aldington and colleagues $^{20}$ recommend that the potential for IFs should be disclosed during informed consent process and the procedure for management of possible findings should be identified. In one of the few studies that have sought research volunteers' opinions, the majority of the volunteers in neuroimaging research expected that their images would be evaluated and medical anomalies disclosed to them. ${ }^{21}$

The balance between the risk and benefit of disclosing unexpected findings depends on the clinical relevance of findings in the diagnosis of asymptomatic disease and the impact of early intervention on outcome. ${ }^{5}$ As majority of IFs may not be clinically relevant, disclosing only relevant findings may be an useful approach. ${ }^{22}$ In the absence of symptomatology, it is uncertain how to reliably determine the relevance of an IF in each research participant. ${ }^{23}$ IFs in this study were classified as clinically relevant independent of the clinical data. Clinically and probably relevant findings were disclosed to the volunteers by the research physician as per departmental standard operating procedure (SOP) on management of IFs. Volunteers were advised to consult a primary care physician for further evaluation. However, clinical outcome of these findings could not be determined as the follow-up was performed independent of the research protocol. The actual impact on the health effects therefore could not be ascertained.

Established mechanisms for handling research IFs vary significantly in different institution or might not exist. ${ }^{24}$ Federal regulations require that consent forms and the consent process address both risks and benefits of the research. Consent forms must describe "any reasonably foreseeable risks" and "any benefits". The continued development of protocols for managing IFs in research and the establishment of guidelines are needed to ensure that research procedures reflect the best interest of participants. $9,25,26$ Such clinical action may provide medical benefit, although, to a small number of research participants.

\section{CONCLUSION}

Since many people with active TB do not experience symptoms at the early stages, there is a possibility that these individuals may not seek medical care at early stage. Passive case-finding therefore may lead to missed or delayed diagnoses for many individuals. ${ }^{27}$ Appropriate diagnosis and early treatment of TB improves health outcome. In one of the systematic reviews, CXR screening had greater accuracy compared to symptom screening. ${ }^{28}$ Therefore, active screening for tuberculosis in selected risk groups in regions with high burden of the disease shall benefit individuals and also public health.

\section{REFERENCES}

[1] Cataldi V, Laporta T, Sverzellati N, et al. Detection of incidental vertebral fractures on routine lateral chest radiographs. Radiol Med 2008;113(7):968-77.

[2] Majumdar SR, Kim N, Colman I, et al. Incidental vertebral fractures discovered with chest radiography in the emergency department: prevalence, recognition, and osteoporosis management in a cohort of elderly patients. Arch Intern Med 2005;165(8):905-9. 
[3] Pickup CM, Nee PA, Randall PE. Radiographic features in 1016 adults admitted to hospital with acute asthma. J Accid Emerg Med 1994;11(4):234-7.

[4] Orme NM, Fletcher JG, Siddiki HA, et al. Incidental findings in imaging research: evaluating incidence, benefit, and burden. Arch Intern Med 2010;170(17):1525-32.

[5] Booth TC, Jackson A, Wardlaw JM, et al. Incidental findings found in healthy volunteers during imaging performed for research: current legal and ethical implications. British Journal of Radiology 2010;83(990):456-65.

[6] Ost D, Fein AM, Feinsilver SH. The solitary pulmonary nodule. N Engl J Med 2003;348:2535-42.

[7] Hall WB, Truitt SG, Scheunemann LP, et al. The prevalence of clinically relevant incidental findings on chest computed tomographic angiograms ordered to diagnose pulmonary embolism. Arch Intern Med 2009;169(21):1961-5.

[8] Gould MK, Fletcher J, Iannettoni MD, et al. Evaluation of patients with pulmonary nodules: when is it lung cancer? ACCP evidence-based clinical practice guidelines (2nd edition). Chest 2007;132(3 Suppl):108S-130S.

[9] Wolf SM, Lawrenz FP, Nelson CA, et al. Managing incidental findings in human subjects research: analysis and recommendations. J Law Med Ethics 2008;36(2):219-48.

[10] Morris Z, Whiteley WN, Longstreth WT, et al. Incidental findings on brain magnetic resonance imaging: systematic review and meta-analysis. BMJ 2009;339:b3016.

[11] Morin SH, Cobbold JF, Lim AK, et al. Incidental findings in healthy control research subjects using whole-body MRI. Eur J Radiol 2009;72(3):529-33.

[12] Jacobs PC, Mali WP, Grobbee DE, et al. Prevalence of incidental findings in computed tomographic screening of the chest: a systematic review. J Comput Assist Tomogr 2008;32(2):214-21.

[13] Vugt SV, Broekhuizen L, Zuithoff $\mathrm{N}$, et al. Incidental chest radiographic findings in adult patients with acute cough. Ann Fam Med 2012;10(6):510-5.

[14] Bass JR, Farer LS, Hopewell PC, et al. American thoracic society. Diagnostic standards and classification of tuberculosis. Am Rev Respir Dis 1990;142(3):725-35.

[15] Korzeniewska KM, Krysl J, Muller N, et al. Tuberculosis in young adults and the elderly: a prospective comparison study. Chest 1994;106(1):28-32.
[16] Work related chronic obstructive pulmonary disease (COPD). In Great Britain in 2014. Available at www.hse.gov.uk/statistics/causdis/copd.

[17] Becklake MR. Occupational exposures: evidence for a causal association with chronic obstructive pulmonary disease. Am Rev Respir Dis 1989;140(3 Pt 2):S85-91.

[18] Balmes J, Becklake M, Blanc P, et al. American thoracic society statement: occupational contribution to the burden of airway disease. Am J Respir Crit Care Med 2003;167(5):787-97.

[19] Blanc PD, Torén K. Occupation in chronic obstructive pulmonary disease and chronic bronchitis: an update. Int J Tuberc Lung Dis 2007;11(3):251-7.

[20] Aldington S, Shirtcliffe P, Nowitz M, et al. Incidental findings from lung CT scans: implications for research. J Med Imaging Radiat Oncol 2011;55(1):20-5.

[21] Kirschen MP, Jaworska A, Illes J. Subjects' expectations in neuroimaging research. J Magn Reson Imaging 2006;23(2):205-9.

[22] Illes J, Desmond JE, Huang LF, et al. Ethical and practical considerations in managing incidental findings in functional magnetic resonance imaging. Brain Cogn 2002;50(3):358-65.

[23] Miller FA, Giacomini M, Ahern C, et al. When research seems like clinical care: a qualitative study of the communication of individual cancer genetic results. BMC Med Ethics 2008;9:4.

[24] Illes J, Kirschen MP, Karetsky K, et al. Discovery and disclosure of incidental findings in neuroimaging research. J Magn Reson Imaging 2004;20(5):743-7.

[25] Wolf SM, Paradise J, Caga-anan C. The law of incidental findings in human subjects research: establishing researchers duties. J Law Med Ethics 2008;36(2):36183.

[26] Cramer SC, Wu J, Hanson JA, et al. A system for addressing incidental findings in neuroimaging research. Neuroimage 2011;55(3):1020-3.

[27] Systematic screening for active tuberculosis: principles and recommendations. World Health Organization 2013.

[28] Van't Hoog AH, Langendam MW, Mitchell E, et al. A systematic review of the sensitivity and specificity of symptom- and chest-radiography screening for active pulmonary tuberculosis in HIV-negative persons and persons with unknown HIV status. WHO 2013. 